\title{
Principles of Operation of a DNP Prepolarizer Coupled to a Rodent MRI Scanner
}

\author{
A. Comment ${ }^{1}$, B. van den Brandt ${ }^{2}$, K. Uffmann ${ }^{3}$, F. Kurdzesau ${ }^{1,2}$, \\ S. Jannin ${ }^{1}$, J. A. Konter ${ }^{2}$, P. Hautle ${ }^{2}$,W. T. Wenckebach ${ }^{1}$, \\ R. Gruetter ${ }^{3}$, and J. J. van der Klink ${ }^{1}$ \\ ${ }^{1}$ Laboratory for Physics of Nanostructured Materials, \\ Ecole Polytechnique Fédérale de Lausanne, Lausanne, Switzerland \\ ${ }^{2}$ Paul Scherrer Institute, Villigen, Switzerland \\ ${ }^{3}$ Laboratory for Functional and Metabolic Imaging, \\ Ecole Polytechnique Fédérale de Lausanne, \\ Lausanne, Switzerland
}

Received 22 October 2007; revised 25 January 2008

(C) Spinger-Verlag 2008

\begin{abstract}
A dynamic nuclear polarization prepolarizer was developed and coupled to a $9.4 \mathrm{~T}$ rodent magnetic resonance imaging scanner in order to perform in vivo hyperpolarization experiments. In the present paper, emphasis is put on methods and hardware performance rather than on in vivo results obtained with this setup. An overview of the main hardware components is given. The full procedure starting from the sample preparation and solid-state polarization to in vivo infusion is described.
\end{abstract}

\section{Introduction}

Because it is a noninvasive versatile technique, magnetic resonance (MR) has become an essential tool in biomedicine. In particular, ${ }^{13} \mathrm{C}$-label $\mathrm{MR}$ spectroscopy experiments are widely used to investigate brain metabolism in vivo. However, the intrinsic low sensitivity of the technique limits the study to highly concentrated chemicals. Hyperpolarization by means of dynamic nuclear polarization (DNP) provides a way to increase the signal of tracers by several thousandfold [1]. For instance, the infusion of DNP-enhanced labeled molecules allows for the measurement of low ${ }^{13} \mathrm{C}$ concentration in tissues. The aim of the setup presented in this paper is to polarize the nuclei of molecules of metabolic interest and to deliver them in a reproducible manner to a living animal inside a rodent-type magnetic resonance imaging scanner. 


\section{System Overview}

The system is based on a continuous-flow cryostat designed at the Paul Scherrer Institute that fits into a standard wide-bore nuclear magnetic resonance (NMR) magnet. The base temperature of this cryostat is below $1 \mathrm{~K}$. The cooling procedure from 300 to $1 \mathrm{~K}$ takes 3 to $4 \mathrm{~h}$ and requires 5 liters of liquid $\mathrm{He}$; the overall consumption during a typical week of experiment is about 60 liters. A schematic representation of the cryostat is shown in Fig. 1. The principle of the cryostat is based on a toroidal phase separator which collects the liquid helium withdrawn from a storage dewar through an entry port by means of a membrane pump connected to an output port. The helium gas which evaporates in the phase separator is evacuated via the membrane pump. A capillary carries liquid helium from the phase separator to the sample space which is kept at a pressure of less than $0.1 \mathrm{kPa}$ by a Roots pump (flow rate, $250 \mathrm{~m}^{3} / \mathrm{h}$ ) backed by a rotary vane pump (flow rate, $65 \mathrm{~m}^{3} / \mathrm{h}$ ). The aperture of the capillary is adjusted by a needle valve remotely controlled from the top of the cryostat.

The field of a $7 \mathrm{~T}$ superconducting magnet was set to $3.35 \mathrm{~T}$ in order to perform DNP at $94 \mathrm{GHz}$. The DNP cavity is designed such as to contain large samples. Since the requirement for DNP experiments is to saturate the electron spin resonance (ESR) transitions everywhere in the sample, a multimode cavity with an extremely low quality factor is used so that the excitation field is relatively homogeneous over the whole sample. It simply consists of a brass cylinder opened at the top. A sample holder with a net volume of $0.6 \mathrm{ml}$ is placed in

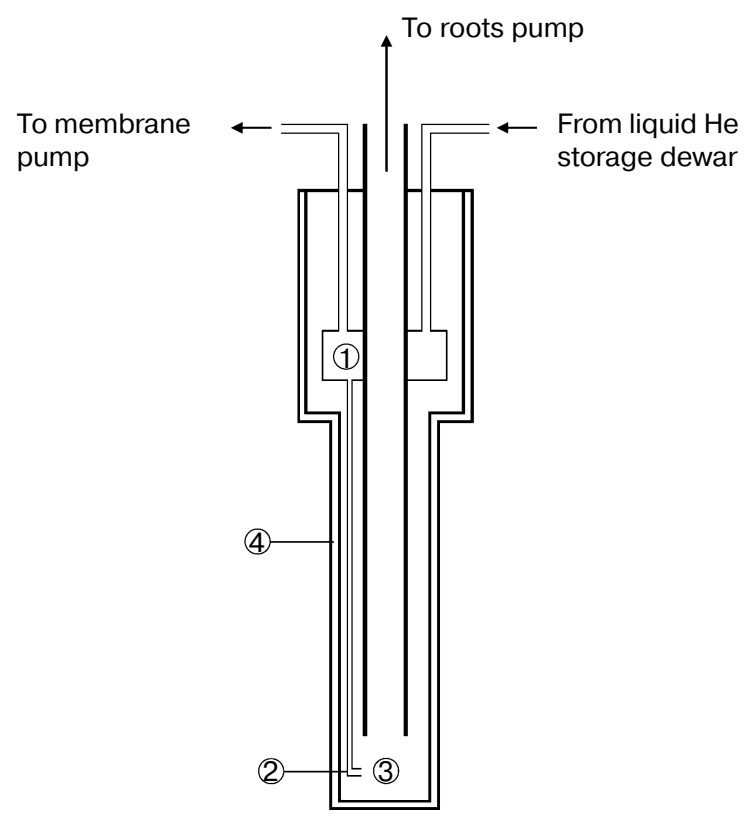

Fig. 1. Sketch of a cross-sectional view of the continuous-flow cryostat: 1, toroidal phase separator; 2 , capillary with needle valve; 3 , sample space; 4 , vacuum mantle. 


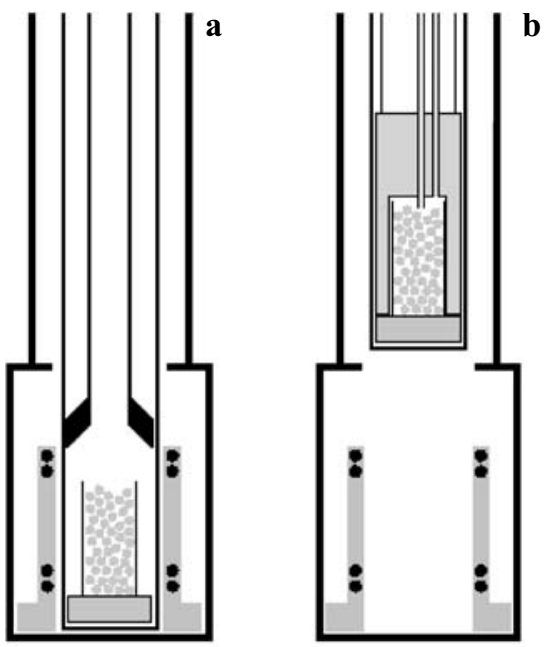

Fig. 2. Sketch of a cross-sectional view of the microwave insert. The thick lines represent the microwave cavity and its sustaining rods. The sample represented by a collection of small circles is located in a cylindrical sample holder which is placed in a glass fiber tube. The eight dark circles symbolize the NMR saddle coil. a The microwave waveguide ended with a horn is placed in the glass fiber tube for DNP. b For dissolution, the waveguide is replaced by another insert. The hot solvent is projected on the sample through an inlet capillary tube and the dissolved solution is collected through an outlet capillary tube.

a glass fiber tube that can be lifted in and out of the cavity. A saddle coil used to pick up the solid-state NMR signal is fixed on a polytetrafluoroethylene (PTFE) cylinder with an inner diameter slightly larger than the glass fiber tube (Fig. 2). The microwaves are directed on the sample from the cylindrical waveguide through a conical transition forming a "horn antenna" located inside the microwave cavity. Typically, the reflected power is less than $1 \%$ of the transmitted power. Note that, except for the sustaining rods and the coaxial cables (not shown in Fig. 2), the entire microwave insert has full axial symmetry. The design and realization of the insert are therefore rather simple and the space available for the sample can be maximized since there is no microwave coupling structure on the cavity walls.

The solid-state sample dissolution procedure follows the principles described by Ardenkjær-Larsen et al. [1]. The main difference in the configuration described above lies in the fact that during microwave irradiation the waveguide is located on the insert main axis, just above the sample. Therefore, it is necessary to remove the cylindrical waveguide prior to placing the dissolution insert (Fig. 2). This operation takes only a few seconds and consequently its influence on the solid-state nuclear polarization prior to dissolution is negligible since the spinlattice relaxation times are still fairly long at $4.2 \mathrm{~K}$. The dissolution insert outlet is directly connected to an infusion device (see below) placed inside the imager bore, next to the animal. For more technical details on the full system the reader is referred to ref. 2 . 
$\mathbf{a}$

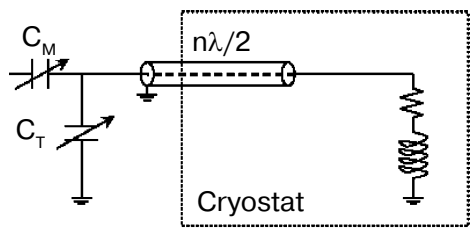

b

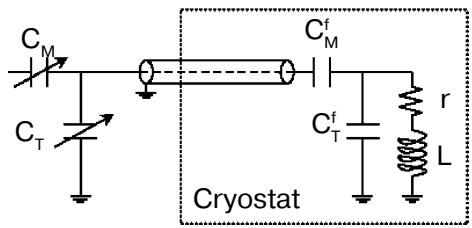

Fig. 3. Remotely tuned and matched low-temperature NMR circuits. $L$ is the inductance of the sample coil and $r$ is its resistance. $C_{\mathrm{M}}$ and $C_{\mathrm{T}}$ are the capacitance values of the room-temperature matching and tuning variable capacitors, respectively. a Standard circuit. b Scheme using two fixed capacitors $\left(C_{\mathrm{M}}^{\mathrm{f}}\right.$ and $\left.C_{\mathrm{T}}^{\mathrm{f}}\right)$ to roughly match and tune the circuit at low temperature. The fixed capacitors are from American Technical Ceramics (type $100 \mathrm{~B}$ ).

\section{Solid-State NMR Measurement}

It is of major importance to accurately evaluate the solid-state nuclear polarization prior to dissolution in order to check the sample quality and to estimate the loss of polarization in the dissolution process. The easiest implementation of a low-temperature NMR probe is to place an NMR coil in the cryostat and to remotely tune and match the resonance circuit with two capacitors located outside the cryostat (Fig. 3a). The coil is connected to the capacitors via a coaxial cable of length $n \lambda / 2$, $\lambda$ being the wavelength corresponding to the Larmor frequency of the monitored nuclei and $n$ being an integer. The problem with such a circuit is its low sensitivity due to the large losses in the coaxial line connecting the coil to the capacitors. The standard solution to this problem would be to place variable capacitors next to the coil, but the lack of space and the very low temperature environment complicate the implementation of such a circuit. An elegant solution is to place small-size fixed capacitors next to the coil to obtain a roughly tuned and matched circuit inside the cryostat and to add a pair of room-temperature variable capacitors on the other end of the coaxial line to fine-tune and match the probe (Fig. 3b). This scheme, which minimizes the radio frequency (RF) losses arising from multiple reflections between the coil and capacitors, was proposed and tested by Conradi [3]. The drawback is that it is a narrow-banded circuit since the fixed capacitors determine the tuning. With this scheme applied to ${ }^{13} \mathrm{C}$ in the system described above, the length of a $5^{\circ}$ tipping pulse was measured to be 500 ns with $200 \mathrm{~W}$ RF power and the single-shot thermal-equilibrium signal-to-noise ratio, defined as the signal amplitude to root-mean-square noise ratio, was on the order of 200 after a $30^{\circ}$ pulse in a typical $3 \mathrm{M}{ }^{13} \mathrm{C}$-labeled sodium acetate sample of $0.4 \mathrm{ml}$ at $1.2 \mathrm{~K}$. 


\section{DNP with Nitroxyl Radicals}

Although with this setup DNP can be performed with any type of polarizing agent, the focus was put on the stable nitroxyl radical TEMPO (2,2,6,6-tetramethylpiperidine-1-oxyl). It has the advantage of being widely available and it has a low toxicity, which is, of course, a requirement for in vivo applications [4]. A typical sample consists of a solution containing a molecule of biological interest dissolved in a water-ethanol or water-glycerol mixture doped with TEMPO. Little droplets of the solution are dropped into liquid nitrogen to obtain frozen beads with a diameter of about 1 to $2 \mathrm{~mm}$ and with a homogeneous distribution of TEMPO. The beads are then loaded into the cryostat. Polarization values on the order of $8 \%$ were obtained on ${ }^{13} \mathrm{C}$ in various molecules with less than $30 \mathrm{~mW}$ microwave power. The typical TEMPO concentration is around $33 \mathrm{mM}$.

One important consideration should be borne in mind when performing DNP with TEMPO, or, more generally, with a radical with a wide ESR line: it is essential to keep the sample temperature as low as possible. Indeed, it was observed experimentally that increasing the temperature from 1.2 to $1.6 \mathrm{~K}$ leads to a polarization decrease of about $25 \%$. This is in agreement with the Borghini model and the related considerations described in ref. 2. Therefore, it is of great importance to work at a low bath temperature, i.e., in a well-designed cryostat coupled to a high-flow pumping system, and consequently it is essential to work with a microwave structure leading to minimal sample heating and to minimize thermal losses through RF connections by using stainless steel coaxial cables.

\section{Strategies for In Vivo Applications with High Turnover}

The short lifetime of the hyperpolarized state of the molecule due to the finite spin-lattice relaxation times of the nuclear spins implies that it is of the utmost importance for in vivo applications to administrate the solution to the animal as quickly as possible. A first obvious requirement is to position the prepolarizer as close as possible to the imager. In the present case, the DNP setup is within 4 $\mathrm{m}$ of the Varian/Magnex actively shielded $9.4 \mathrm{~T}$ imager. Additionally, a specific procedure was developed to shorten the delay between dissolution and in vivo infusion: the liquefied solution is transferred into a remotely controlled plastic infusion device located inside the imager bore through a PTFE capillary tube by high-pressure helium gas. A sketch of the infusion device is shown in Fig. 4. The liquid sample is separated from the gas inside the infusion device before the solution is infused. The procedure is computer-controlled and the typical dissolution and transfer time is $5 \mathrm{~s}$ ( $1 \mathrm{~s}$ for dissolution and $4 \mathrm{~s}$ to push the sample into the imager bore). An additional $1 \mathrm{~s}$ is added as a buffer time before starting the infusion. An optical safety device checks for the presence of gas at the infusion port of the device. A more detailed description of this device is given in ref. 2 . Such a device is necessary to perform experiments in a narrow-bore imager since there is no access for a bolus injection directly into a vein of the animal, but more generally it also increases the level of reproducibility of in vivo experi- 


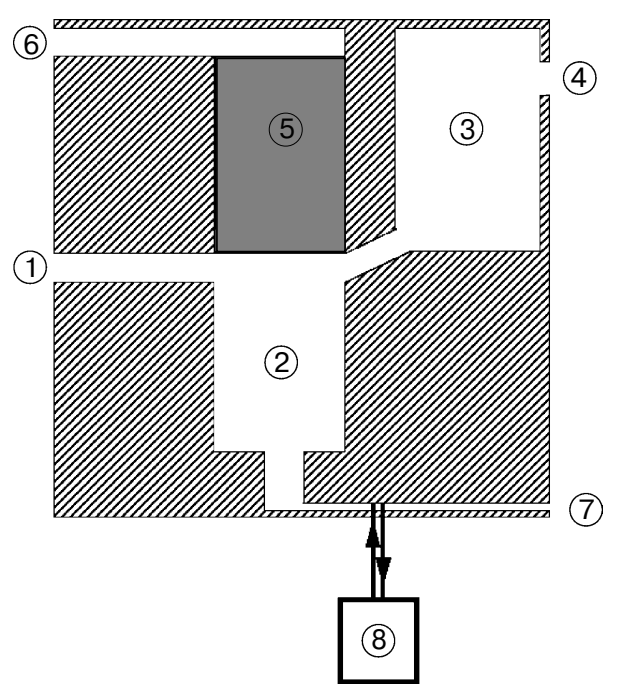

Fig. 4. Schematic representation of the infusion device: the hyperpolarized solution pushed into the device through the inlet port (1) by pressurized He gas is collected in the central compartment (2) while the gas escapes through the vent port (4) located above the overflow chamber (3). Once the pressurized gas flow is stopped, the displacement piston (5) is moved downwards by injecting water through the displacement port (6). Subsequently, the central compartment becomes isolated from the inlet port and the overflow chamber, and the solution is infused through the infusion port (7). An optical device (8) checks for the potential presence of gas at the infusion port and stops the infusion if a gas bubble of nonnegligible size is detected.

ments. In particular, the delay between dissolution, infusion and data acquisition is completely identical for all experiments.

The amount of polarization lost between the dissolution and the infusion depends on the room-temperature $T_{1}$ of the nuclei of interest, which in turn depends on the dissolution solvent and the polarizing agent concentration. A volume of $5 \mathrm{ml}$ of deuterated water heated to $170{ }^{\circ} \mathrm{C}$ is used to dissolve a sample of about $0.4 \mathrm{ml}$, corresponding to a dissolution factor on the order of 15 . Such a low dissolution factor allows for the production of highly concentrated hyperpolarized solutions with a polarizing agent concentration that does not strongly decrease the nuclear spin-lattice relaxation times in a large range of molecules. The turbulences created by the pressurized gas in the central compartment of the infusion device (Fig. 4) force the solution to mix. After the $1 \mathrm{~s}$ buffer time, the homogeneity of the solution is expected to be high. On top of the expected loss calculated from the room-temperature nuclear spin-lattice relaxation time, an additional $10-20 \%$ loss in polarization is typically observed, the source of which has not yet been clearly identified.

The amount of time needed to obtain a solid-state nuclear polarization close to the maximum in a typical sample doped with TEMPO is usually on the order of 30 to $60 \mathrm{~min}$. The time course of a typical in vivo DNP experiment is as follows: loading a new sample takes 5 min; refilling the cryostat with liquid 
helium and measuring a reference signal at $4.2 \mathrm{~K}$ takes approximately $20 \mathrm{~min}$ (the NMR circuit properties do not substantially vary between 1.2 and $4.2 \mathrm{~K}$ and the thermal-equilibrium signal at $4.2 \mathrm{~K}$ can be used to evaluate the nuclear polarization enhancement factor); polarizing the sample at $1.2 \mathrm{~K}$ takes 30 to $60 \mathrm{~min}$; finally the dissolution and the infusion procedures take $5 \mathrm{~min}$. Therefore, the total time of a full experiment is approximately one to one and a half hour. The amount of liquid helium requested for such an experiment is about 3 liters.

\section{Conclusions}

By using a versatile and low-consumption cryostat and the widely available TEMPO free radical, it was possible to rapidly polarize nuclei in various compounds via DNP with low microwave power levels. For in vivo applications, a fast transfer and infusion procedure based on a remotely controlled infusion device was developed to minimize the delay between sample dissolution and infusion and thus to minimize the polarization loss in the hyperpolarized solution. The setup was used for the infusion of DNP-enhanced molecules into animals placed in a narrow-bore high-field imager. To this day, many successful in vivo experiments have been performed using this setup and the results will be published elsewhere.

\section{Acknowledgments}

This work is supported by the Swiss National Science Foundation, grant 200021109479, by the Fund of the Dean of the Faculty of Basic Sciences, Ecole Polytechnique Fédérale de Lausanne (EPFL) and by the Paul Scherrer Institute. We thank the Centre d'Imagerie Biomedicale (CIBM) of the Université de Lausanne, Université de Genève, EPFL, Centre Hospitalier Universitaire Vaudois and Hôpitaux Universitaires de Genève for access to its facilities. The CIBM is supported by the Jeantet and Leenaards Foundations.

\section{References}

1. Ardenkjaer-Larsen, J.H., Fridlund, B., Gram, A., Hansson, G., Hansson, L., Lerche, M.H., Servin, R., Thaning, M., Golman, K.: Proc. Natl. Acad. Sci. USA 100, 10158-10163 (2003)

2. Comment, A., van den Brandt, B., Uffmann, K., Kurdzesau, F., Jannin, S., Konter, J.A., Hautle, P., Wenckebach, W.T., Gruetter, R., van der Klink, J.J.: Concepts Magn. Reson. 31B, 255-269 (2007)

3. Conradi, M.S.: Concepts Magn. Reson. 5, 243-262 (1993)

4. Ankel, E.G., Lai, C.-S., Hopwood, L.E., Zivkovic, Z.: Life Sci. 40, 495-498 (1987)

Authors' address: Arnaud Comment, IPN-SB, Ecole Polytechnique Fédérale de Lausanne, station 3, 1015 Lausanne, Switzerland

E-mail: arnaud.comment@epfl.ch 